\title{
Meteor studies applying incoherent scatter radar instruments
}

\author{
Ingrid Mann ${ }^{1}$, Asta Pellinen-Wannberg ${ }^{2}$ and Anders Tjulin ${ }^{3}$ \\ ${ }^{1}$ EISCAT Scientific Association, Kiruna and Umea University, Sweden \\ email: ingrid.mann@eiscat.se \\ ${ }^{2}$ IRF Kiruna and Umea University, Umea, Sweden, ${ }^{3}$ EISCAT Scientific Association, Kiruna, \\ Sweden
}

\begin{abstract}
One of the interesting aspects of optical meteor studies is that the spectral composition of the brightness reveals information about the element composition of the solid particles that enter Earthfs atmosphere from interplanetary space. Deriving composition from optical spectra requires understanding the entry process during which the entering solid interacts with atmospheric species. This is especially so, because most meteors are observed at altitudes where the mean free path changes from tens of meters to millimeters, that is in the $120 \mathrm{~km}$ to $80 \mathrm{~km}$ altitude range within the atmosphere. The ionization that causes optical emission also reflects radio waves, so that meteors are observed with different kinds of radar instruments. Incoherent scatter radar facilities are in particular designed to study the upper atmosphere by using the backscattering from free electrons and are basically High Power Large Aperture radars. During the past 15 years they have been increasingly used for meteor studies. The phased-array incoherent scatter radars that are currently under development, such as the planned EISCAT3Dsystem in northern Scandinavia, will further improve the spatial and time resolution of radar observations and will allow simultaneous measurements of the meteors and of the parameters of the surrounding ionosphere. Radar backscattering is also sensitive to objects that are smaller than those detected optically, so that the observations also permit studying the extension of the meteoroid size distribution to smaller sizes. In this presentation we consider the possibilities for measurements with the future EISCAT-3D as a new path of studying the physics of meteor phenomena with high accuracy.
\end{abstract}

